\title{
Gold, Cryptocurrency and Oil Adaptive Analysis and Forecasting
}

\author{
Ilvira Khasanova ${ }^{1},{ }^{*}$ Lubov Orlik $^{2}$, Galina Zhukova ${ }^{1}$ \\ ${ }^{1}$ Financial University Under The Government Of The Russian Federation, Russia \\ ${ }^{2}$ Russian State Social University, Russia \\ *Email: ilvira.1996@mail.ru
}

\begin{abstract}
The work is devoted to identifying and tracking development trends, structural shifts in the economy under the influence of world markets, represented by non-stationary time series of gold, bitcoin and oil prices. The heuristic potential of the concept of the long and medium wave is used for forecasting purposes. The analysis of financial time series using the adaptive correlation coefficient is carried out. The dynamics of the traditional coefficient appears to be a significantly smoothed graph, which prevents sufficient qualitative analysis of the data. The results obtained are analysed to identify wave fluctuations, to determine the phases of growth, prosperity, recession and stagnation in the economy. An overview of the situation on the world markets for gold, bitcoin and oil based on the considered time series is presented. Based on the identified trends in the dynamics of these markets, short-term forecasting was carried out using ARIMA models and neural networks. The statistical calculations $\mathrm{R}$ environment is used.
\end{abstract}

Keywords: Juglar cycles, Adaptive correlation coefficient, Cryptocurrency, Gold, Oil.

\section{INTRODUCTION}

The ideas of the cyclical development of the economy are reflected in the chrono-economic concepts. Periodic fluctuations in total economic activity, depending on the duration, are divided into short-term, which include Kitchin cycles, medium-term, for example, the Juglar cycles, and long-term, which include the Kuznets, Kondratiev and others cycles. Cycles consist of four recurring economic phases - rise, prosperity, decline, stagnation, as well as four borderline situations between these phases. Since the cycles are considered as an essential component of the economic environment, they will be observed both at the level of the main macroeconomic indicators and the level, for example, of stock indices, exchange rates $[1,2]$. Technical analysis of time series relies on data on the mass behaviour of factors in the foreign exchange and resource markets. By identifying trends in the dynamics of these markets, it is possible to carry out short and medium-term forecasting.

\section{AN OVERVIEW OF THE GOLD, CRYPTOCURRENCY AND OIL VOLATILITY}

From the financial instrument point of view, gold is the most proven and stable asset during any economic crisis, which forms its favourable price conjuncture in the market. Gold is considered both an investment asset and a luxury item at the same time, which contributes to its demand around the world, but reduces its correlation with other assets. Historical data on gold prices show that its value is subject to fluctuations, and there is no upward trend over a relatively long time. Also, gold prices depend on the industrial demand of the jewellery, electrical and other industries, on the economic and political state of the leading countries of the world.

Cryptocurrency is a digital currency with decentralised accounting and issuance (excludes a single centre for issuing payment units), operating based on Blockchain technology. Each operation is divided between the parties to the transaction and recognised as 


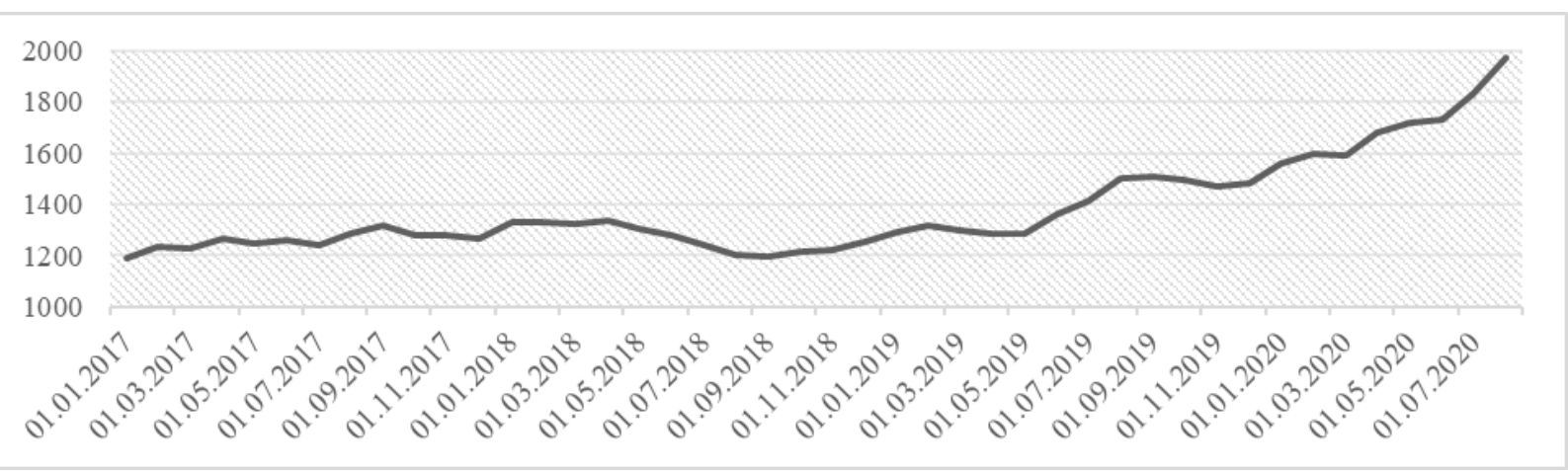

Figure 1 Dynamics of prices per ounce of gold in US dollars. Source: Quandl. Retrieved from: https://www.quandl.com/ (access date 01.08.2020).

valid only after confirmation by other users. A distinctive feature of this technology is the preservation in the form of a register of all financial transactions carried out without identifying financial agents performing transactions. Cryptocurrency rates are confirmed by high volatility; this is partly since the market appeared not so long ago and is distinguished by a small number of participants and trading volumes. The market determines the cost of a cryptocurrency, that is, the more demand, the higher its rate. In turn, the demand depends on the advantages that cryptocurrency has to fiat money, on its popularity, publicity, on the government's policy towards it and legislative decisions, political events. The most popular cryptocurrency currently is Bitcoin, created in 2008 by a man under the pseudonym Satoshi Nakamoto. Bitcoin emerged in the midst of a global crisis. In August 2020 , bitcoin capitalisation reached $\$ 226$ billion. This shows that Bitcoin can compete with most fiat currencies in assessing the volume of the cash supply.

Gold and cryptocurrency have several similar characteristics: in contrast to fiat currencies, they have limited emission, and their production (mining) process requires not only financial but also resource costs [3]. During the period of deteriorating market conditions, they are considered as a tool for hedging risks; therefore, during periods of economic, social and political shocks and crises, their prices change in the same way. If we compare the dynamics of gold prices and the BTC rate against the dollar in Figures 1 and 2, it can be noted that starting from 2019, a similar dynamics of rates has been observed. Against the background of the strengthening of the trade confrontation between the United States and China, the introduction of new trade duties, prices rise until August, and in August-September, they reach their maximums for the year. Prices are falling in the fourth quarter. In early 2020, the conflict between Iran and the United States escalated, which led to an increase in demand for gold, as a result of which prices reach their peak values and continue to rise. The coronavirus epidemic broke out in China, which triggered the entry of the world economy into the global crisis and led to high volatility in all markets. At the same time, the main mining capacities are concentrated in China. In March, prices for both gold and bitcoin are declining, but starting from April, they begin to rise. In May, the third halving of bitcoin took place, that is, the reward for adding a new block was halved, which had a favourable effect on its value. The instability of the economic situation, political and economic risks, for example, the US presidential elections in 2020, stimulate investors to turn to the metals and cryptocurrency markets.

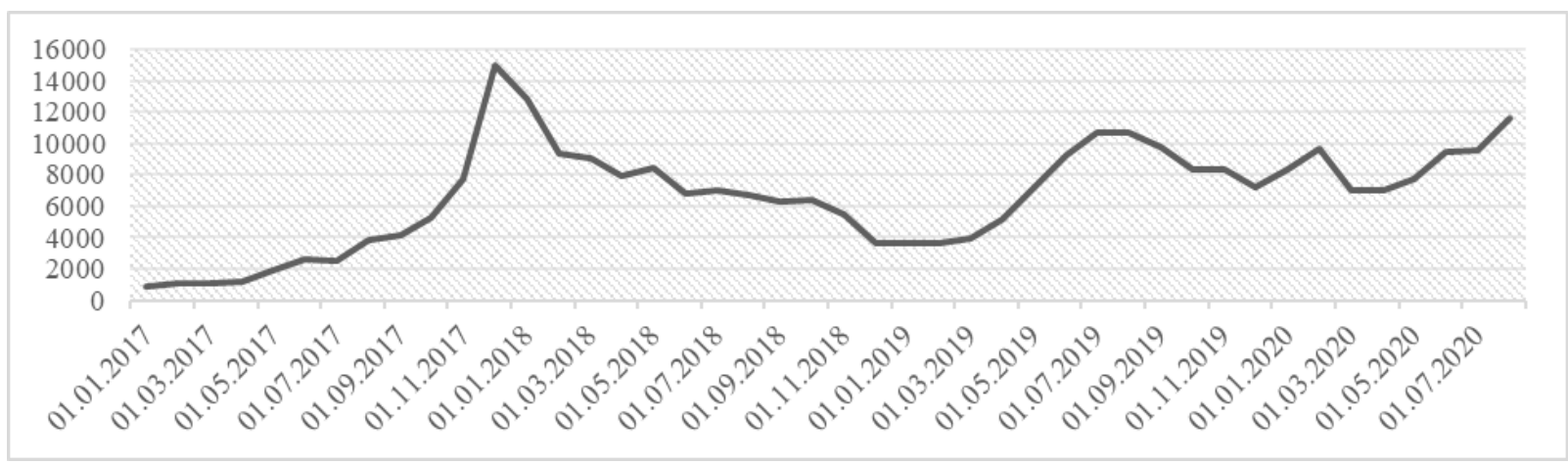

Figure 2 Dynamics of the BTC to USD exchange rate. Source: Quandl. Retrieved from: https://www.quandl.com/ (access date 01.08.2020). 
One of the conditions for the stability and competitiveness of the economies of many countries is oil. Its limited world reserves determine the generated supply for this raw material. The price level is directly influenced by the behaviour of OPEC - the organisation of oil-exporting countries. The mission of the organisation is to stabilise oil markets by setting quotas for oil production. The price is also influenced by political factors and the demand changes under the influence of various macroeconomic indicators. In the long term, oil prices will decline due to the gradual transition to environmentally sustainable energy sources [4].

\section{ANALYSIS OF FINANCIAL TIME SERIES USING THE ADAPTIVE CORRELATION COEFFICIENT}

In [5], using the example of currency quotes, it is shown that, despite similar values, the adaptive coefficient is more sensitive than the modified correlation coefficient. Let's analyse the financial time series using an adaptive correlation coefficient. As a statistical base, we will take data on the cost of an ounce of gold, bitcoin cryptocurrency and one barrel of Brent oil. The adaptive correlation coefficient allows us to identify the dynamics of the considered time series [6,7].

$$
\begin{aligned}
& r_{t}(\alpha)=\frac{s_{t}}{d_{t}} \\
& s_{t}=(1-\alpha) s_{i-1}+\alpha\left(\Delta x_{1 t} \Delta x_{2 t} \ldots \Delta x_{i t}\right), t= \\
& 1,2, \ldots, T \\
& d_{t}=(1-\alpha) d_{i-1}+\alpha\left|\Delta x_{1 t} \Delta x_{2 t} \ldots \Delta x_{i t}\right|, \\
& 0<\alpha<1
\end{aligned}
$$

$s_{t}$ and $d_{t}$ - exponentially weighted moving averages of the products of increments and absolute products of increments of two series, $\alpha$ - smoothing constant or adaptation parameter can be selected from the specified range $[0,1]$, based on the specific application of the calculated correlation coefficient.

Figure 3 shows the resulting plot of the adaptive correlation coefficient [8,9]. The considered time is divided into periods. In the first period (from 2016 to mid-2017), the coefficient shows an average negative relationship. The beginning of the medium-term Juglar cycle [2] can be attributed to this period. Indeed, the relationship between gold, bitcoin and oil is being tracked in world markets. At the end of June 2016, a referendum was held, as a result of which the majority supported the UK's withdrawal from the European Union. In anticipation of this event, bitcoin prices have increased. In July, another halving of bitcoin took place, which also served as a factor in the growth of prices. The course was influenced by the tightening of regulation of cryptocurrencies in China, political instability, conflicts in Donbass, and the presidential elections in the United States in November. In 2017, the bitcoin rate continued to grow. In September, the People's Bank of China, by its actions, brought down the BTC rate. Still, in October the cryptocurrency recovered its positions, continued to grow and reached its record values in December. The gold rate in 2016 is growing until November while reaching its maximum value in July. In 2017, there was a variety of trends. As a result of the agreement of the OPEC countries to reduce oil production, world consumption increased, and prices for it began to rise from the second half of 2016. Along with the rise in Brent oil prices, the US-Canadian shale sector began to develop. This growth continued in 2017.

In the second selected period (from mid-2017 to mid2019), the adaptive correlation coefficient changes in the range $[-0.2 ; 0.2]$. During this period, due to the numerous coefficient values changes, the connection between all the considered series is absent. The period from 20182019 is the flourishing phase of the Juglar cycle. In February 2018, the bitcoin rate fell. This was due to the

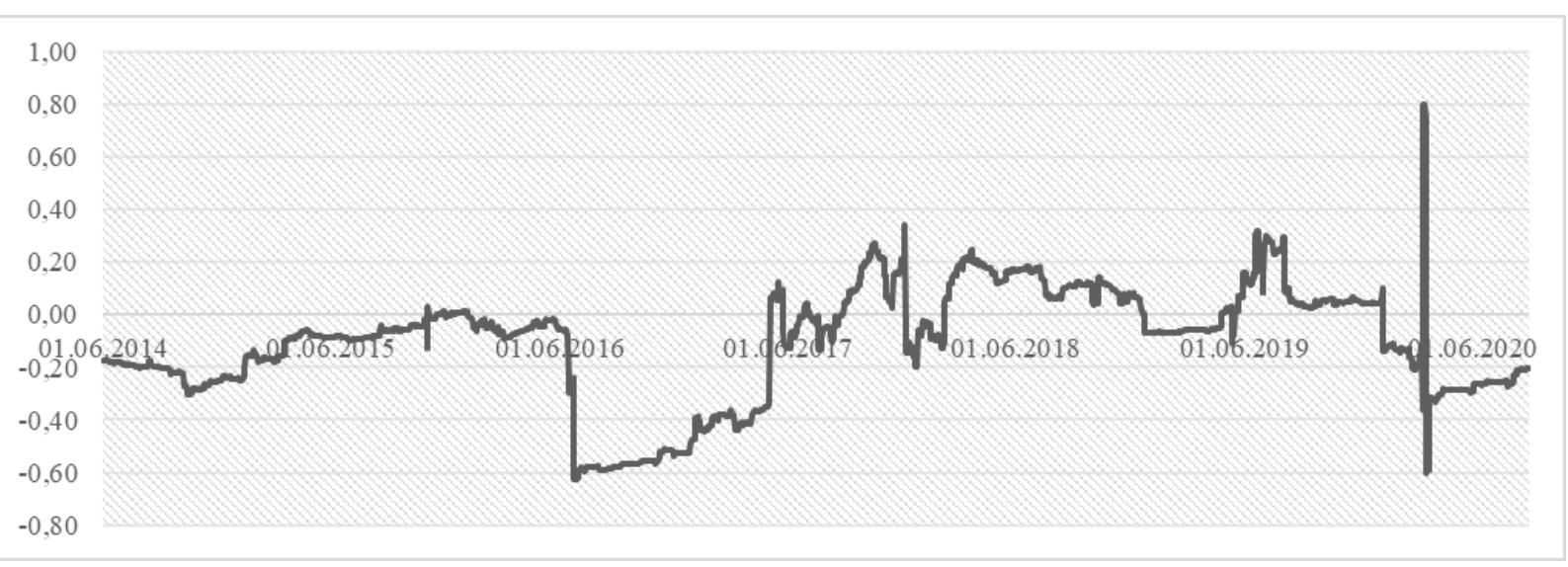

Figure 3 Graph of the adaptive correlation coefficient of gold, prices for 1 barrel of Brent oil and BTC cryptocurrency (calculated by the author based on data from Quandl and World Bank Open Data sources). 
ban on cryptocurrency advertisements by Facebook, Google and Twitter. By mid-December, the rate fell by almost $80 \%$ relative to the previous year. Due to the fall in the rate, miners began to close their businesses. Due to the strengthening of the US dollar, the gold rate is falling. Oil prices continue to rise and peak in early October. This was facilitated by the expectation of a reduction in Iran's oil exports since November due to US sanctions and an increase in global demand for oil. However, the imposed sanctions against Iran did not lead to such serious supply cuts as expected, and at the same time, the growth in demand of the largest oil consumers - China and India slowed down. After the quotes moved to a fall, which lasted until December.

In the third period (mid-2019 - early 2020), the correlation coefficient shows an average positive relationship. In the next period, there is a sharp jump in the coefficient. The prosperity phase of the Juglar cycle occurs before the beginning of 2020, after which the recession phase begins. In 2019, bitcoin futures were launched; this type of trading has gained popularity. At the same time, there was an increase in the cryptocurrency rate, the peak of growth fell in July, and then the price decreased throughout the year. Gold in dollars managed to reach new highs, the highest price was in September. In July 2019, the OPEC member countries extended the agreement to limit oil production. Oil prices rose sharply at the end of September following an attack on two major oil facilities in Saudi Arabia. Success in trade negotiations between the United States and China contributed to the rise in prices.

The sharp jump in the fourth period is due to the onset of a pandemic. 2020 was marked by the entry of the world economy into a global crisis, the trigger of which was the coronavirus. The result is a sharp drop in demand for oil and oil products. Only in China - by $20 \%$ even though this country provided $2 / 3$ of the world demand for oil. There was a sharp drop in prices due to the lack of an agreement between Russia and OPEC. The overflow of vaults and the closure of futures led to the fact that WTI crude oil fell to negative values. At the same time, earlier, the netback price for Russian oil was also negative when prices in Europe were lower than the cost of transporting oil from Russia to Europe. Prices recovered in May after a record fall in April. After the price decline in March, both bitcoin and gold rates continue to rise. Instability in the world provokes market participants to transfer funds to reserve assets.

\section{ANALYSIS OF FINANCIAL TIME SERIES IN THE R SOFTWARE ENVIRONMENT}

To plot the corresponding graphs of changes in the rates of gold and bitcoin (figure 4.1. - 4.2.) the plot function was used. When constructing all the graphs to obtain financial and economic data in real-time, the data library - Quandl was used. For time series forecasting, ARIMA models (figure 5.1 - 5.2) and neural networks (figure 6.1 - 6.2) were used. The ARIMA model combines the two most widely used approaches to forecasting time series: autoregressive - the value of the series is linearly dependent on the previous values, and the moving average - information about the entire history of the series is concentrated in the model errors in previous periods. The neural network after training can predict the future value of a sequence of exchange rates based on previous values, thanks to the new experimental function for forecasting "nnetar", which implements the ability of the neural network to generalise and highlight the hidden relationships between input and output data $[10,11]$.

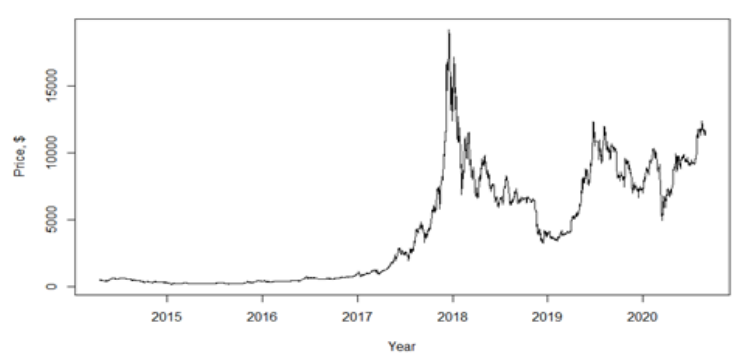

Figure 4.1. BTC rate time series.

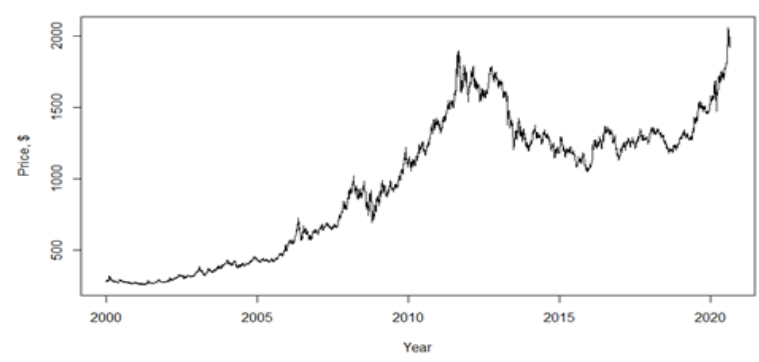

Figure 4.2. Time series of gold price (ounce).

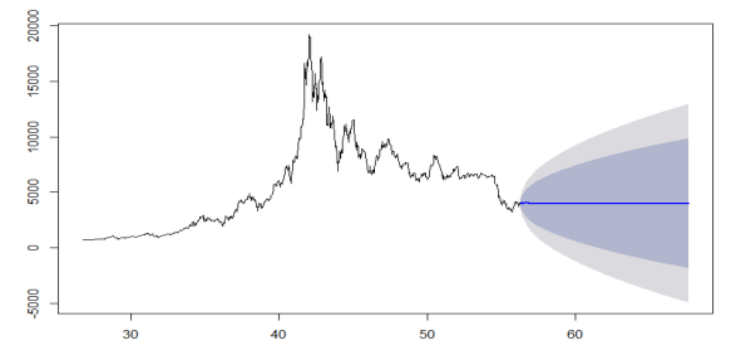

Figure 5.1. Forecasting BTC rate using ARIMA model

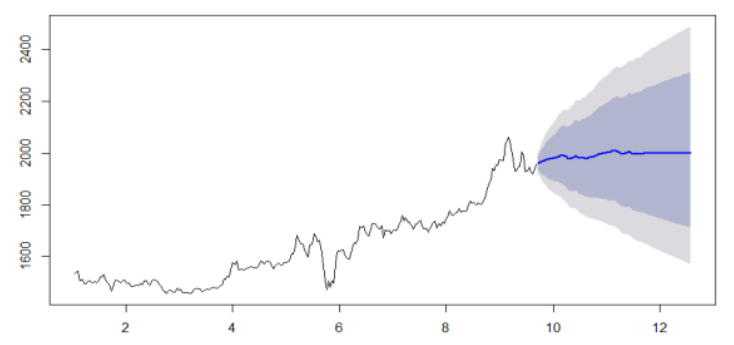

Figure 5.2. Forecasting the gold rate using the ARIMA model. 


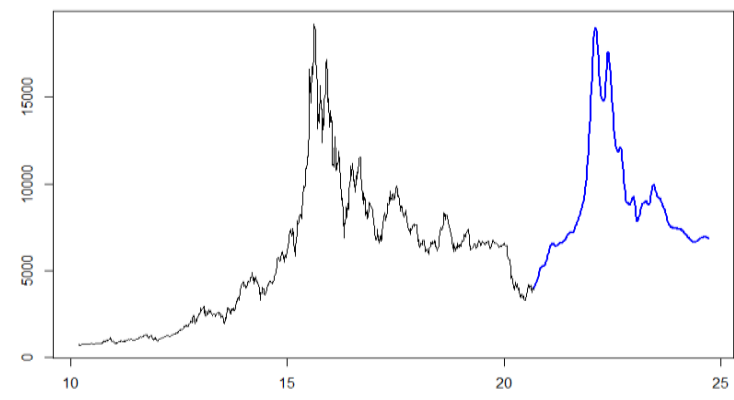

Figure 6.1. Forecasting BTC rate using a neural network model.

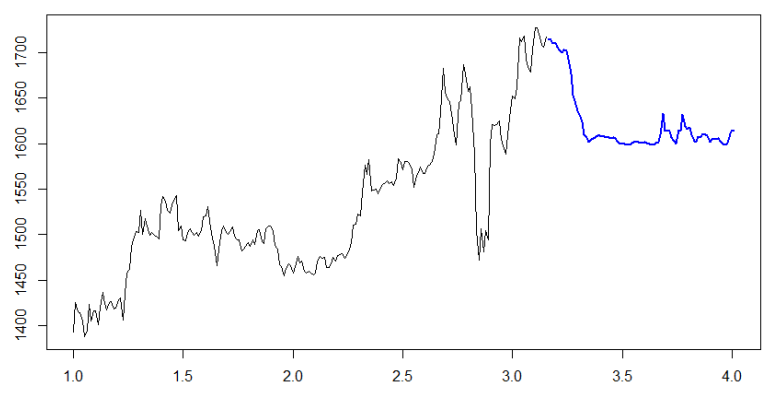

Figure 6.2. Forecasting gold rate using a neural network model.

The basis for the bitcoin rate building models was the time series from $01 / 01 / 2014$ to $12 / 01 / 2019$, and the forecast was made from $12 / 01 / 2019$. The basis for the gold rate building models was the time series from $01 / 01 / 2014$ to $12 / 31 / 2019$, and the forecast was made from 01/01/2020. Due to insufficient data on the time series of the bitcoin course for training the model, ARIMA did not show any changes. The neural network copied the last pattern. For the gold rate, the ARIMA model predicts small abrupt deviations and rate stability, since a constant level of the process is observed throughout the entire forecasting area of the currency pair. In the neural networks model, two periods can be distinguished: the first is a sharp decrease in the exchange rate, and the second is the stabilisation of the series. It is better to use a neural network model for forecasting, because the forecast accuracy is higher compared to the ARIMA model, and the average absolute percentage error is less. It is also worth considering that forecasting is influenced by many factors that cannot be taken into account, for example, political ones.

According to the graphs obtained, a fall in the rates of Bitcoin and gold is predicted. The work [5] shows the analysis of the movement of oil prices and their fall in the long term. The behaviour of all the variables under consideration is similar; accordingly, when analysed, the adaptive correlation coefficient will show a strong positive relationship. In this case, the forecast period falls into the stage of stagnation of the Juglar cycle in the economy, which means that the dynamics of changes in these markets fit into the dynamics of the phases of the Juglar cycles.

\section{CONCLUSIONS}

The adaptive correlation coefficient of time series is calculated using the example of the dependence of the gold currency pair, BTC cryptocurrency and Brent oil prices. The analysis of the movement of exchange rates and oil prices in the software environment R. Short-term forecasts were built in the R environment using ARIMA models and a neural network. Using non-traditional correlation analysis, the relationship between the studied series is shown, as well as high sensitivity to changes in the studied variables of the adaptive correlation coefficient. When analysing the graphs obtained using the adaptive correlation coefficient, the phases of recovery, prosperity, recession and stagnation in the economy are shown. The dynamics of price changes in the gold, cryptocurrency and oil markets fit into the dynamics of the phases of Juglar cycles. When the economy is on an upward wave of the cycle, prices rise and are at a high level. When the world economic situation deteriorates, they are at a low stationary level. The forecasts obtained also correspond to the phases of the Juglar cycles.

\section{REFERENCES}

[1] N.D. Kondratiev, Big cycles of conjuncture and the theory of foresight, Moscow: MFK, 2002, pp. 341401.

[2] A.A. Akaev, Big cycles of the conjuncture and the innovation-cyclical theory of economic development of Schumpeter-Kondratiev, Economic science of modern Russia 2(61) (2013) 7-29.

[3] M.A. Magomedov, A.M. Alikberova, Bitcoin: essence, mechanism of operation and genesis of cryptocurrency, Bulletin of the Dagestan State University, Series 3: Social Sciences, 2018. DOI: https://doi.org/10.21779/2500-1930-2018-33-2-49$\underline{54}$

[4] Yu.N. Bobylev, The World Oil Market: Price Stabilization, Economic Development of Russia, Moscow: Foundation "Economic Policy Institute named after E.T. Gaidar" Vol. 26 Iss. 7 (2019) 29 31 .

[5] L.K. Orlik, I.F. Khasanova, Dynamics of Exchange Rates and Oil Price: Adaptive Analysis and Forecasting, International Conference on Economics, Management and Technologies, 2020, pp. 229-234.

DOI: https://doi.org/10.2991/aebmr.k.200509.042

[6] Yu. Lukashin, Adaptive methods of short-term forecasting of time series, Finance and Statistics, 2003, p. 413. 
[7] G. Zhukova, D. Kagirova, Adaptive approach to the analysis of correlation properties of financial time series, 2018, pp. 1-5. DOI: https://www.researchgate.net/publication/3292921 10_Adaptive_approach_to_the_analysis_of_correla tion_properties_of financial_time_series

[8] World Bank Open Data Retrieved from: https://data.worldbank.org/ (date accessed: 30.04.2020).

[9] Quandl Retrieved from: https://www.quandl.com/ (date accessed 01.08.2020).

[10] P.B. Kratovich, Neural Network Model for Forecasting Time Series of Financial Data, International Journal Software Products and Systems 1 (2010) 132-135.

[11] D. Kagirova, Forecasting the dynamics of quotations of currency pairs on the basis of $r$ : ARIMA and the neural network model, "The Concept" Scientific and methodical electronic journal Vol. 39 (2017) 3581-3585. 